\title{
SPECTRAL AND TEMPORAL BEHAVIOR OF AN ERBIUM DOPED FIBER LASER
}

\author{
E. LACOT, F. STOECKEL and M. CHENEVIER \\ Laboratoire de Spectrométrie Physique, BP. 87, Université Joseph Fourier, \\ F-38402 Saint Martin d'Hères cedex, France
}

\begin{abstract}
The dynamics of an erbium doped fiber laser are analysed under continuous and modulated pumping. It is found that the bondaries between different modes depend only on the pump parameter and the lifetime of the photon in the cavity. Spectral and temporal behaviors are analysed.
\end{abstract}

\section{Introduction}

Fiber lasers are potential laser sources for optical coherent communication (1) We believe that these lasers could also be used in spectroscopy with their wavelength region in the mid infra-red (2)(3) or in the visible range by up conversion (4). They have low threshold and can be pumped with diode lasers.

Unfortunately fiber lasers exhibit high nonlinearity leading to spontaneous relaxation. Our aim is to use a fiber laser for intracavity laser absorption spectroscopy (ICLAS) (5). In this technique, the spectro-temporal dynamics play an important role. We present here the spectral and temporal behavior of such a laser.

\section{Experimental}

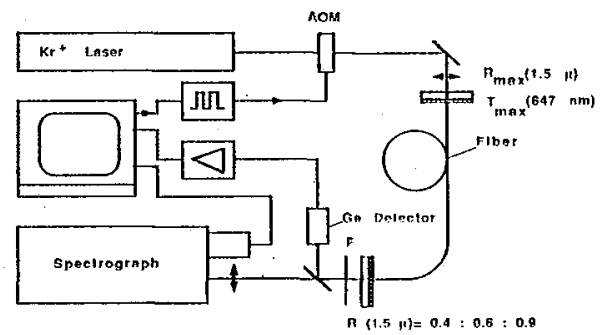

Fig.1 Schematic diagram of the experiment.

Figure 1 shows the experimental arrangement of the erbium laser pumped by a krypton ionized laser. Throughout this work, the pump wavelength was fixed at $647 \mathrm{~nm}$ which corresponds to the transition ${ }^{4} \mathrm{~F} 9 / 2 \leftarrow$ ${ }^{4} \mathrm{I}_{15 / 2}$. The pump beam passes through an acousto-optic modulator (AOM). In cw pumping, the AOM is used as a variable attenuator. The intensity of the first order beam can then be changed without any angular deviation, which usually happens when the krypton laser power is monitored electrically. Such procedure avoids for example false hysteresis in the fiber laser power / pump laser power curve due to small angular deviation combined with thermal effects. In the study of the dynamic behavior, the AOM. is used to modulate the pump power. The pump beam is coupled into the erbium doped fiber through a dichroic mirror. The distance between the dichroic mirror and the entrance to the fiber can be altered from a position of contact to a separation of a few microns. This space acts like a Fabry Perot and can be used to select the wavelength of the laser.

The output mirror is butt-coupled with the fiber. The reflection coefficient $R$ of the output mirror can be changed from 0.4 to 0.9 . The erbium-doped fiber (provided by CNET) has a concentration of $12010^{-6}$, a core radius of $6.3 \mu \mathrm{m}$ and a length changing from 0.5 to $3 \mathrm{~m}$. The fiber is single mode at the laser operating 
wavelength $(\lambda=1.5 \mu)$ and is pumped with a maximum pumping power of $900 \mathrm{~mW}$. The lifetime of the ${ }^{4} \mathrm{I}_{13 / 2}$ level was measured and found to be equal to $12 \mathrm{~ms}$.

The fiber laser beam is split and sent into a $60 \mathrm{~cm}$ monochromator and to a high speed germanium detector for the temporal behavior. All the signals are digitalized and stored by means of a computer. For the purpose of the temporal study, the injected pump power into the fiber laser is modulated at different frequencies and different pump modulations.

\section{Experimental method}

\subsection{Determination of the characteristics of the laser}

When the $\mathrm{Kr}^{+}$pump is applied to the fiber laser as a step function, the fiber laser beam shows, after a starting time $t_{t h}$, an exponential decaying oscillation with a period $\Theta$, followed either by a stable output in the $\mathrm{cw}$ mode or by an increasing oscillation which reaches a stationary amplitude, depending on the pump rate and the photon lifetime in the cavity.

In this first stage (exponential decay), the laser can be described by a simple three level system. The time needed to reach the threshold is then given by (6):

$$
t_{t h}=\frac{\tau}{\omega_{\mathrm{p}} \tau+1} \ln \left[\frac{2 \omega_{\mathrm{p}} \tau . \mathrm{NBT}}{\omega_{\mathrm{p}} \tau(\mathrm{NBT}-1)-(\mathrm{NBT}+1)}\right]
$$

$\mathrm{N}$ : is the total population, B: the Einstein coefficient, $T$ : the lifetime of the photon in the cavity, $\tau:$ the lifetime of the upper level, $\omega_{\mathrm{p}}$ : the pump rate $=\sigma_{\mathrm{p}} I-\mathrm{P}_{\mathrm{p}}, \sigma_{\mathrm{p}}$ : the pump cross section, $\mathrm{I}_{\mathrm{p}}$ : the pump photon density, $\mathrm{P}_{\mathrm{p}}$ : the krypton pump power, while the period of the oscillation $\Theta$ is given by :

$$
\frac{1}{\Theta^{2}}=\frac{(\mathrm{NBT}-1) \omega_{\mathrm{p}}-(\mathrm{NBT}+1)}{(2 \pi)^{2} \tau \mathrm{T}}
$$

Figure 2 gives a typical initial behavior when the laser is working in the $\mathrm{cw}$ mode and in the relaxation mode.

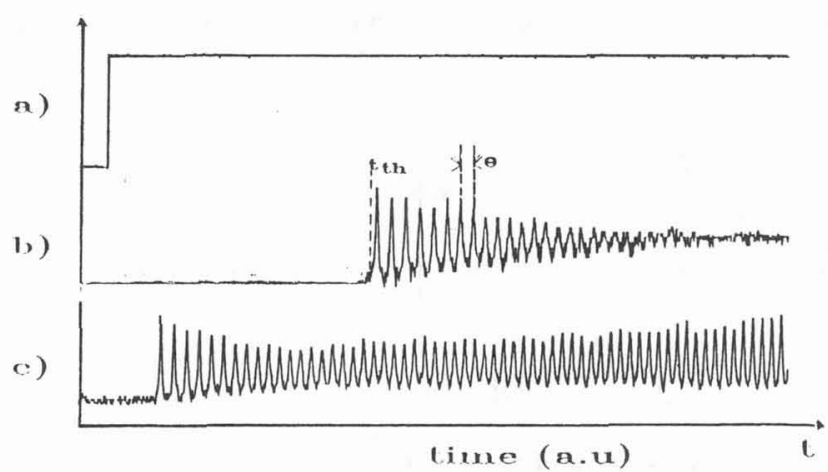

Fig.2 Starting behavior of the fiber laser
a) $\mathrm{Kr}^{+}$pump beam
b) fiber laser in the $\mathrm{cw}$ mode
c) fiber laser in the relaxation mode.

Figure 3 shows the experimental curve of the starting time $t_{\text {th }}$ versus the krypton pump power, the solid line gives the best fit with the equation (1). This allows us to determine the pump rate $\omega_{p}$ and the quantity NBT. Figure 4 shows the inverse of the square of the period $\Theta$ of the exponential decaying oscillation versus the krypton power. By adjusting the equation (2) to data and considering the previous results, we determine the photon lifetime $T$ in the cavity .

The different working regions of the fiber laser can then be represented in a $T / \omega_{p} \tau$ diagram (Fig.5). In the lower left region the laser is below the threshold, in the upper right, the laser is in the free relaxation mode, whilst in the central part the laser is in the $\mathrm{cw}$ domain. The boundary between $\mathrm{cw}$ and the free relaxation domain is interpreted as a Hopf bifurcation (7). 


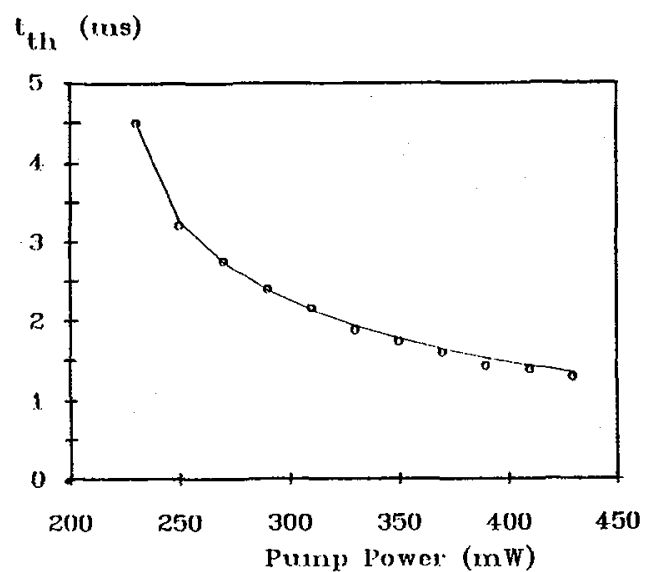

Fig.3 Time needed to reach the threshold th versus the krypton pump power.

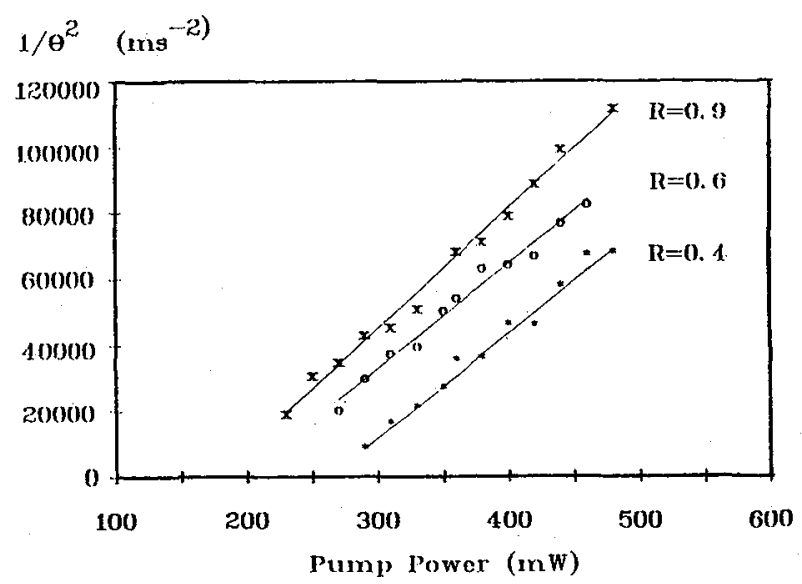

Fig.4 Inverse of the square of the period $\Theta$ of the oscillation versus the krypton pump power.

\section{2. cw mode}

In the cw mode, the threshold of the pump power is typically about $200 \mathrm{~mW}$ (measured at the exit of the krypton laser). Figure 6 shows the cw output power at $1.5 \mu$ versus the pump power for three different output couplers.

'T' (photon lifetime) (ns)

Output Power (mw)

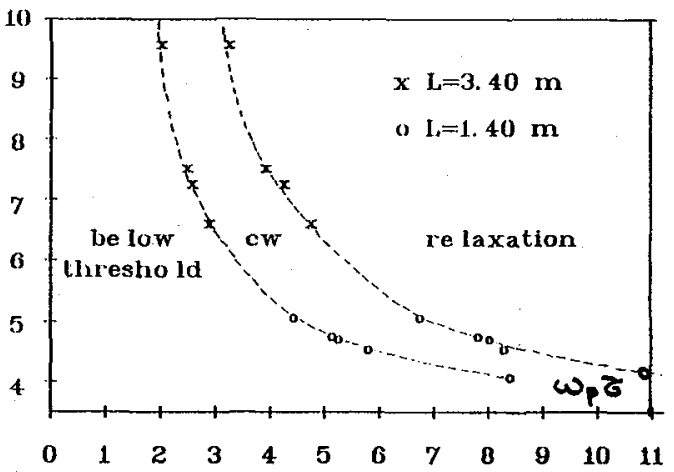

Fig. 5 Hopf bifurcation diagram showing the below threshold, cw, and relaxation region of the fiber laser.

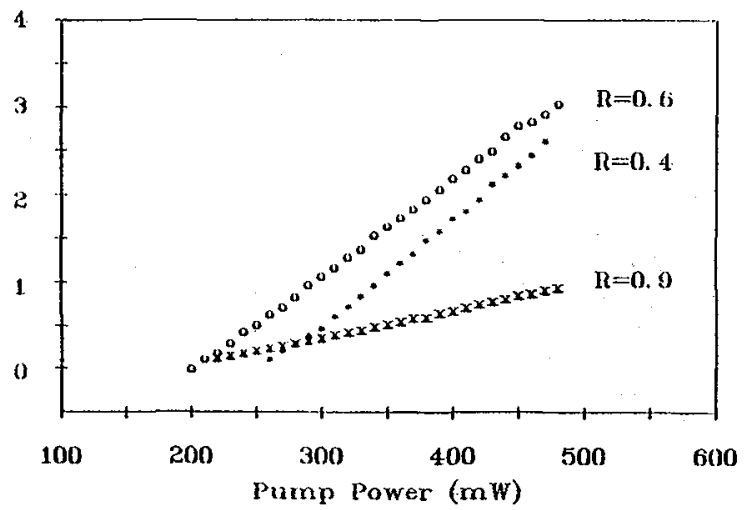

Fig. $6 \mathrm{cw}$ output versus the krypton pump power.

\subsection{Free relaxation mode}

As it was shown in Figure 5, by increasing the pump power, or by increasing the photon lifetime in the cavity (by a better alignment or by butt coupling the input mirror), the laser begins to operate in the free relaxation region.

The only selective element comes from the low finesse Fabry Perot effect due to the small distance $d$ between the input mirror and the fiber entrance. By slightly changing this distance with a PZT translator, the wavelength of the fiber laser can be adjusted to the first gain peak at $\lambda_{1}=1.538 \mu$ or to the second one at $\lambda_{2}=1.55 \mu$. The low finesse Fabry Perot is not selective enough to isolate only the $\lambda_{2}$ wavelength. Figure 7 depicts the temporal and the spectral evolution for three different pump powers with two different adjustments of the distance $d$.

It should be noted that when the fiber laser is operating solely at the wavelength $\lambda_{1}$, by increasing the pump power, the laser passes from the $\mathrm{cw}$ to the relaxation domain; for higher pump power the wave shape of the intensity of the fiber laser is asymmetric in shape whilst at lower pump power the shape is nearly sinusoidal 
with a continuous baseline. When the laser is operating simultaneously at the two wavelengths $\lambda_{1}$ and $\lambda_{2}$, then in the relaxation mode the wave shape exhibits a double periodicity $\mathrm{T}$ and $2 \mathrm{~T}$.

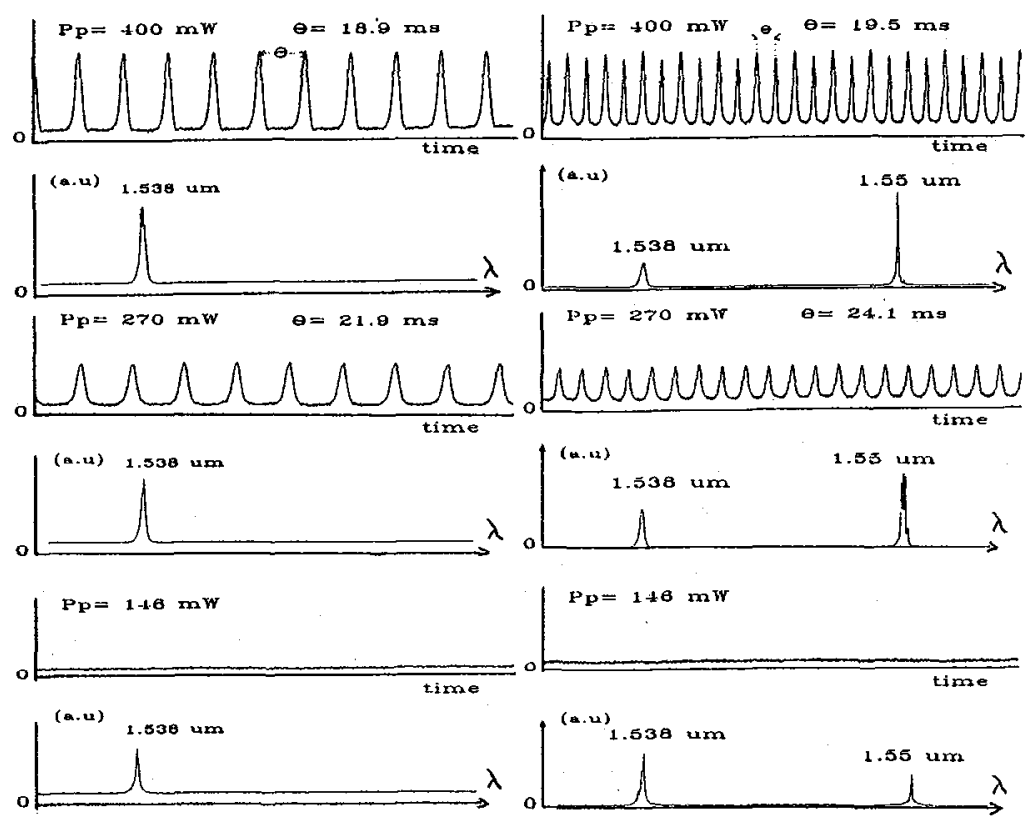

Fig.7 Temporal and spectral evolution in the free run region $\left(\lambda_{1}=1.538 \mu ; \lambda_{2}=1.55 \mu\right)$.

\subsection{External modulation}

Figure 8 shows the response of the fiber laser to a sinusoidal pump modulation for different modulation frequencies. At lower frequencies, the fiber laser follows the modulation whereas for higher frequencies the laser undergoes a cascade of period doubling until a chaotic region (Fig.9). By continuing to increase the frequency modulation, the laser reaches an inverse cascade of period doubling. The number of regions depends strongly on of the pump parameter $\omega_{\mathrm{p}}$, and on the amplitude of the modulation or the lifetime of the photon in the cavity as it is shown in Figure 10.

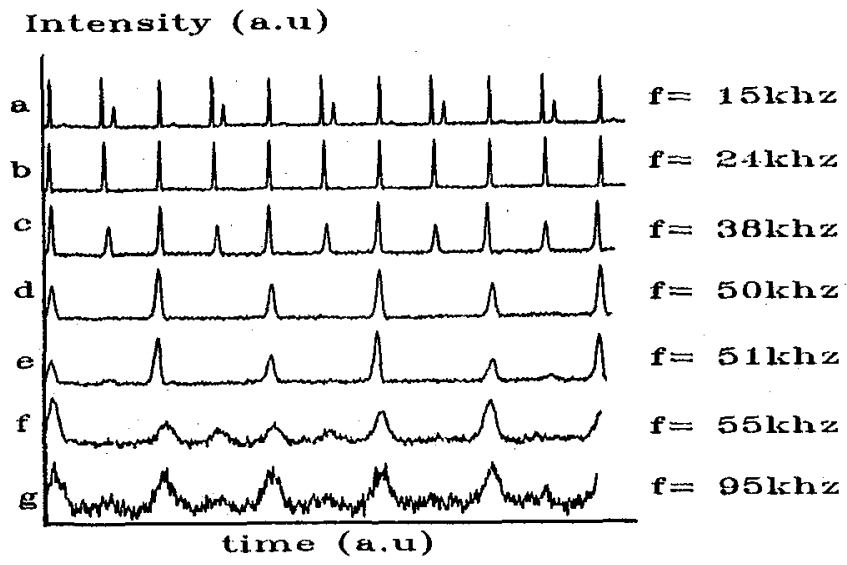

Fig.8 Response of the fiber laser to a periodic pump modulation. 
The bifurcation diagram, like the wave shape, depends on the wavelength of the fiber laser as it is shown in Fig.11. For the same modulation amplitude, a sequence $T, 2 T, T, 2 T, 4 T, 2 T, T$ occurs when only $\lambda_{1}$ exists while the sequence $T, 2 T, T, 2 T, 4 T, 8 T, 4 T, 2 T$, T exists when $\lambda_{1}$ and $\lambda_{2}$ coexist.

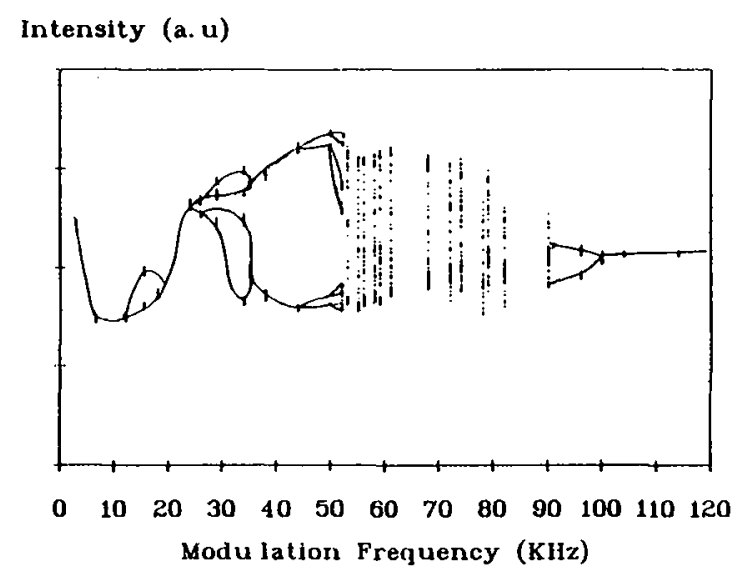

Fig 9 Typical bifurcation diagram schowing the chaotic region

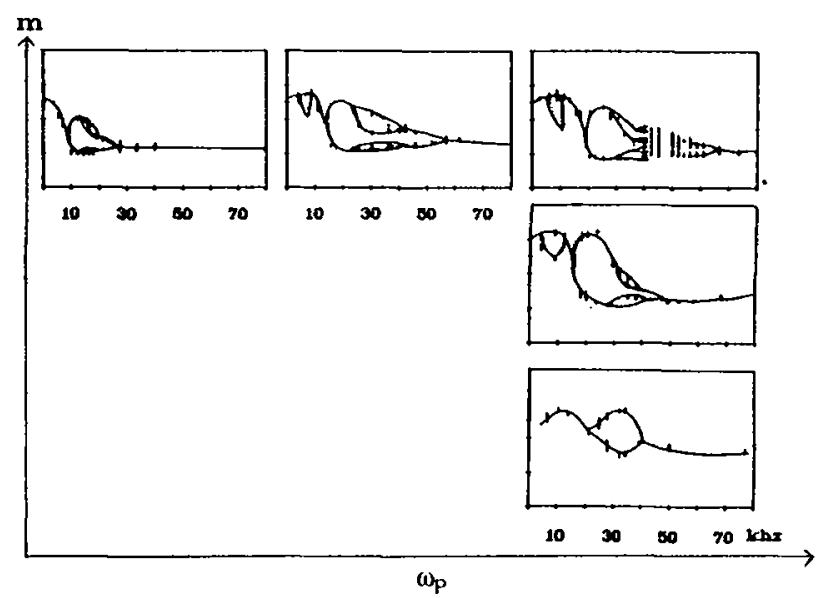

Fig.10 Type of bifurcation diagram in the space.

$\mathrm{m}$ : modulation parameter, $\omega_{\mathrm{p}}$ : pump parameter.

\section{Discussion}

We have analysed experimentally the dynamics of an erbium doped fiber laser under continuous and modulated pumping and we have found that the boundary is easily represented by the space pumping parameter / lifetime of the photon in the cavity. By changing the length of the fiber, we can describe a larger part of the boundary. A spontaneous relaxation mode occurs above a second threshold. This relaxation mode shows two types of wave shape, single periodicity or double periodicity depending if the laser exhibits one or two wavelengths.

When a modulation is applied to the pump, an evolution towards chaos is observed by changing the frequency. No satisfying theoretical model including realistic parameters can presently describe this behavior. For example, a simple 3 levels model gives a bifurcation diagram with realistic parameters but does not show a spontaneous relaxation.

A model including the population, the ficld and the polarisation, describes both behaviors but with unrealistic parameters. Other models are under progress. 
Acknowledgement

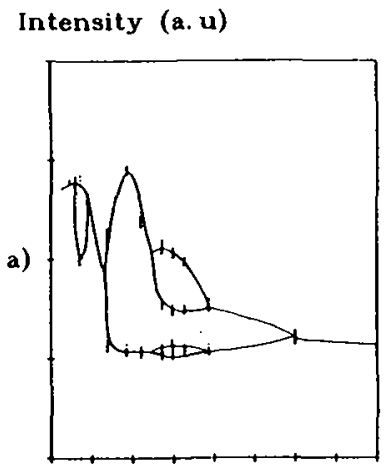

0 1020304050607080
Intensity (a. u)

b)

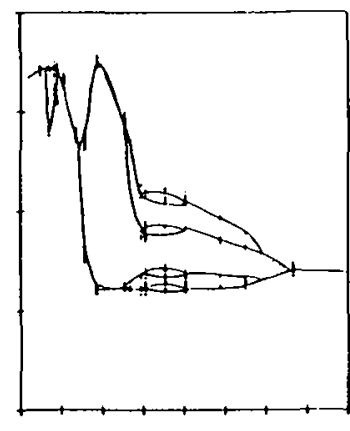

$0 \quad 1020304050607080$ Modu lation Frequency (KHz)

Fig.11 Bifurcation diagrams when the laser is lasing a) at $\lambda_{1}=1.538 \mu$,

b) simultaneously at $\lambda_{1}$ and $\lambda_{2}=1.55 \mu$.

Financial support by the DRET Grant No. 89-270 is gratefully acknowledged.

\section{References}

(1) E. DESURVIRE in Proc. Conference on lasers and electro-optics, CLEO'91, Baltimore, 1991.

(2) K. SMITH, E.H. GREER, R. WYATT, P. WHEATLEY, N.J. DORAN and M. LAWRENCE in Proc. Conference on lasers and electro-optics, CLEO'91, Baltimore, 1991.

(3) P. URQUHART

I. EE Proceeding, 1988, 385-407.

(4) J.Y. ALLAIN, M. MONERIE, H. POIGNANT Electron Lett., 26, 1990,261.

(5) A. CAMPARGUE, F. STOECKEL, M. CHENEVIER Spectrochimica Acta Rev., vol 13, n¹, 1990, 69.

(6) L. TARASSOV

in Physique des processus dans les générateurs de rayonnement optique cohérent. (éd. MIR, Moscow, 1985).

(7) P. BERGE, Y. POMMEAU, Ch. VIDAL

in L'ordre dans le chaos (éd. Hermann, 1984). 